NIST Technical Note 1862

\title{
gtklogger: A Tool For Systematically Testing Graphical User Interfaces
}

\author{
Stephen A. Langer \\ Yannick Congo \\ Andrew C.E. Reid \\ Rhonald C. Lua \\ Valerie Coffman
}

This publication is available free of charge from: http://dx.doi.org/10.6028/NIST.TN.1862

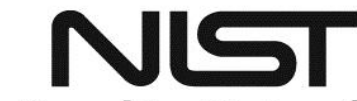

National Institute of Standards and Technology U.S. Department of Commerce 


\title{
NIST Technical Note 1862
}

\section{gtklogger: A Tool For Systematically Testing Graphical User Interfaces}

\author{
Stephen A. Langer \\ Yannick Congo \\ Applied and Computational Mathematics Division \\ Information Technology Laboratory \\ Andrew C.E. Reid \\ Materials Science and Engineering Division \\ Material Measurement Laboratory \\ Rhonald C. Lua \\ Metallurgy Division \\ Materials Science and Engineering Laboratory \\ Valerie Coffman \\ Mathematical and Computational Sciences Division \\ Information Technology Laboratory
}

This publication is available free of charge from:

http://dx.doi.org/10.6028/NIST.TN.1862

August 2015

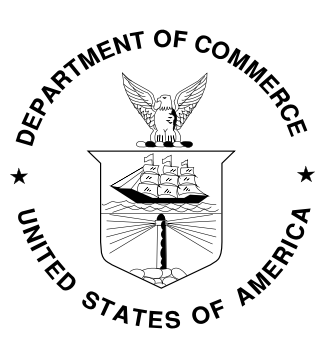

U.S. Department of Commerce

Penny Pritzker, Secretary

National Institute of Standards and Technology Willie May, Under Secretary of Commerce for Standards and Technology and Director 
Certain commercial entities, equipment, or materials may be identified in this document in order to describe an experimental procedure or concept adequately. Such identification is not intended to imply recommendation or endorsement by the National Institute of Standards and Technology, nor is it intended to imply that the entities, materials, or equipment are necessarily the best available for the purpose.

National Institute of Standards and Technology Technical Note 1862

Natl. Inst. Stand. Technol. Tech. Note 1862, 19 pages (August 2015) CODEN: NTNOEF

This publication is available free of charge from: http://dx.doi.org/10.6028/NIST.TN.1862 


\begin{abstract}
We describe a scheme for systematically testing the operation of a graphical user interface (GUI). The scheme provides a capability for generating event logs, which are recordings of a user session with the interface. These logs can be annotated with assertion statements, comparing reference test data with data retrieved by introspection on the GUI elements. Such an annotated log forms a test case, suitable for incorporation into a regression test suite.
\end{abstract}

Keywords: automatic testing, graphical user interface testing, python, gtk 


\section{Introduction}

It is well established among software developers that systematic testing of software, whether against a formal design specification or against a less formal case-by-case assesment of correct functionality, is a critical part of the software development cycle, and crucial to the production of high-quality software.

Much formal testing methodology revolves around viewing software products as essentially functions, in the mathematical sense, abstractly taking a single input from some large domain, performing a computation, and producing a single output. Increases in both the raw power of computers and in the power and scope of software development tools have led to increasing complexity in software. Presenting this complexity to the user in a comprehensible way demands a graphical user interface, which has its own complexity, and makes the software a stateful, interactive machine. While it is still true that the software can be thought of as a function, now the inputs and outputs have to be considered to be the full state of the program and its graphical user interface (GUI). This complicates the application of traditional testing methods. At the same time, the GUI raises the bar for the expected reliability - it allows users more rapid access to potentially-overlooked corner cases, and encourages the expectation that all aspects of the program will "just work".

Contact with formal testing methodologies can be restored by the introduction of systematic ways of testing of a graphical user interface. In this article, we take a step in that direction, describing the construction of a testing kit, that allows a user session with a GUI to be recorded and played back, and that allows the state of the GUI widgets to be queried at repeatable locations within the session, ensuring both correctness and consistency of the GUI state.

For our example, we use the GIMP Tool Kit (GTK) widget set [1], specifically gtk+, version 2.6 or later, and its Python wrappers, PyGTK [2]. We have created a Python module, called gtklogger, which can be used to record, replay, and test a program with a PyGTK user interface. gtklogger was developed specifically to test the OOF [3] project at NIST, but can easily be applied to other PyGTK programs. This paper includes instructions for extending gtklogger to handle PyGTK objects that were not used in OOF.

\section{Functional Overview}

The test kit we are constructing must be able to record and replay a user session by logging events to a file, and later reading that file and recreating the events. The first thing to decide is what kind of events should be logged. One possibility is to track every mouse motion, mouse click, and keyboard button press. However, our goal is not to test the components of a graphics library, but to test a program written to use that library. We assume that the graphics library itself is working correctly. The appropriate domain of the test kit is therefore the interface between the graphics library and the application. This approach has a number of advantages:

1. The number of events is greatly reduced. Moving the mouse into a window and clicking on a button is represented by a single 'click' event, rather than a series like ['enter window', 'move mouse', 'enter button', 'move mouse', 'click'].

2. The recorded events are independent of the position of the graphics windows and even of the position of interface components within the windows. The beneficial consequences of this are that (a) Redesigning the user interface will not necessarily invalidate existing test scripts, (b) Test scripts are portable, since they don't depend on any aspects of hardware, and (c) Test scripts are insensitive to other programs running simultaneously. 
We are not the first to suggest this approach. In particular, Andersson and Bache [4] have proposed a similar scheme, also implemented for PyGTK. Our method operates at a slightly lower level of abstraction than this tool, generating actual Python code during the recording mode which is then run in replay mode. This allows us greater flexibility in manipulating and extending the replay functionality, at the price of a greater sensitivity to architectural changes in the GUI.

The operation of the system is shown schematically in Fig. Error! Reference source not found., which shows the flow of control for both the recording and playback cases.

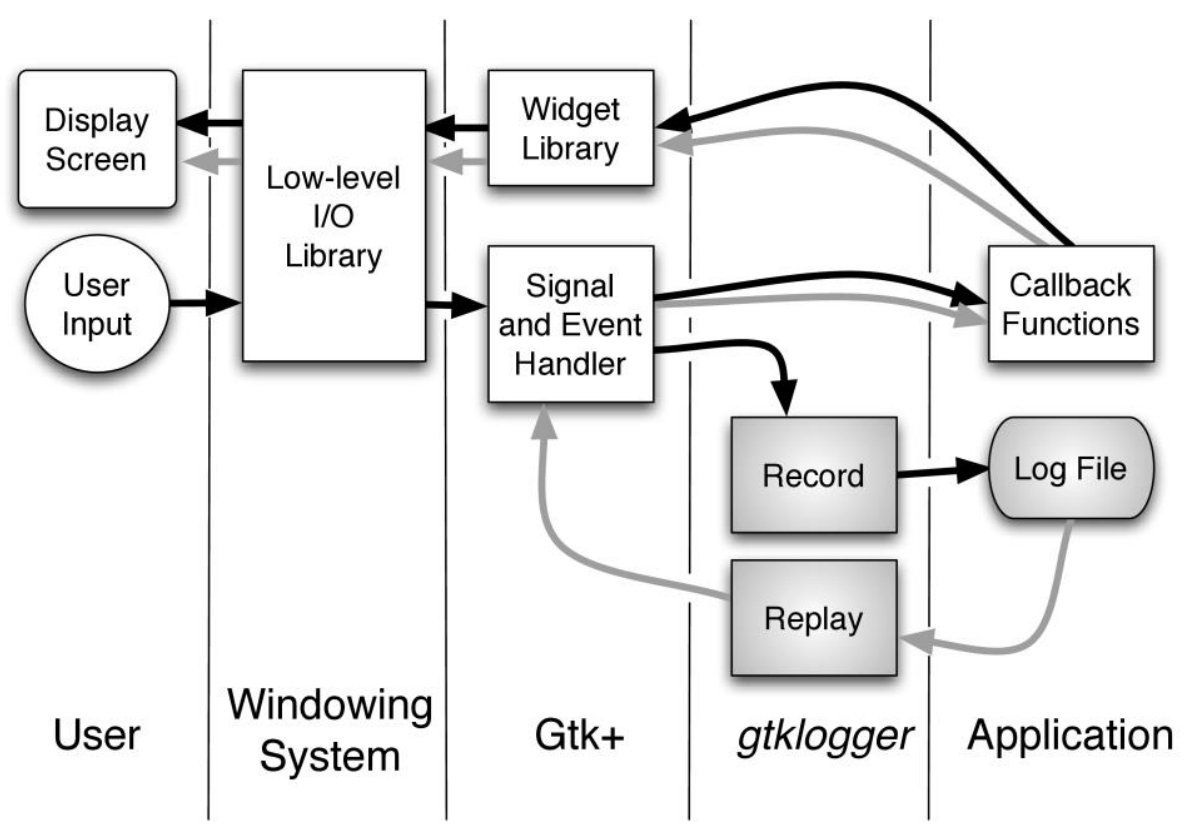

Figure 1: The flow of control during gtklogger operations. Dark arrows show the control path during recording — user input is caught by the windowing system, and generates GTK+ events, which are caught both by the usual callback and by the gtklogger recording system, which generates an entry in a log file, but does not interfere with the execution of the callback. Light arrows illustrate the control path during playback - events are synthesized by the replay mechanism from entries in the log file, and triggers the usual chain of operations, just as though the event had originated with a user.

There are four steps involved in using the gtklogger module to test a PyGTK program. First, the program must be modified ("instrumented") so that it can record events. The gtklogger system provides a substitute for the standard "connect" functionality common to many toolkits, including PyGTK. gtklogger's substitute "connect" function is the hook through which events are recorded. This is described in detail in Sec. 2.1.

Second, the program must be run with recording enabled in order to generate a test case. In the simplest case, this is nearly identical to an ordinary run of the program, the only differences being the modifications to use the gtklogger "connect" scheme, and the setting of a global switch to turn on 
recording. The output of this process is a log file describing all of the recorded events which occurred during the run. Here, user or tester judgement is required to ensure that a useful test case is generated. This is described in greater detail in Sec. 2.2.

Third, the log file should have tests of the program state inserted into it, adding lines of Python code which compare the internal states of program objects, including PyGTK objects, with their correct state for the circumstances. This completes construction of the test case. The gtklogger scheme provides a number of tools for accessing PyGTK objects so that their internal state can be queried, and also additional tools for controlling the order of events in threaded applications. These tools and their use are described in Sec. 2.3.

Finally, the test is implemented by re-running the program, synthesizing the recorded events through the gtklogger replay mechanism, as described in Sec. Table 1: The PyGTK objects that gtklogger currently handles, and the signals that can be logged for each. The list can be extended by defining new GtkLogger subclasses.

3.2. The test fails if any of the assertions in the modified log file raise errors, and passes if they do not. The robustness and suitability of the test is the responsibility of the test case generator. Each of the previous steps need be undertaken only once, but this final step can be treated as in standard regression testing, and re-run whenever the program is modified or ported to a different computer system.

\subsection{Instrumenting a Program}

A gtk+ application consists of a collection of widgets, which are objects (in the object-oriented programming sense) that are drawn on the computer screen and with which the user can interact. Windows, buttons, labels, sliders, and text entry fields are all widgets. Some widgets are containers, with other "child" widgets within them. Containers can be nested, and every visible widget can trace its ancestry through its parent containers back to a top-level widget, which is usually a gtk. Window or gtk.Dialog. Widgets interact with the program by emitting "signals", which are associated with callback functions via a widget's connect method.

When using gtklogger, this basic structure doesn't change, but a few new function calls must be added and a few PyGTK function calls must be replaced by gtklogger variants. The modifications add a small amount of function-calling overhead associated with GUI events in comparison with the uninstrumented code, but in practice, do not affect the execution of the program in any way, except of course when log files are being recorded or replayed. There are a number of ways in which programs must be modified:

1. Widgets must be given identifying names.

2. Signals must be connected to callback functions via the function gtklogger. connect instead of the method gobject. Gobject. connect.

3. Non-widget objects that emit signals must be adopted by named widgets (establishing a parentchild relationship in gtklogger that doesn't necessarily exist in PyGTK).

4. Submenus must be added using the function gtklogger. set_submenu instead of the method gtk.MenuItem.set_submenu.

5. gtk. Dialogs must be replaced by gtklogger. Dialogs.

6. Checkpoints must be inserted.

These operations are described in more detail in the following subsections. 


\subsubsection{Naming Widgets}

When gtklogger is replaying a line from a log file, it has to be able to identify the widget that caused the line to be recorded, in order to recreate the event. gtklogger identifies widgets by giving them names ${ }^{1}$, and keeping track of all of the existing top-level widgets. Because container widgets know their children, knowing the name of a widget and all of its parents enables gtklogger to address the actual widget object. Names of top-level widgets are assigned like this:

window $=$ gtk. Window ( )

gtklogger.newTopLevelWidget (window, 'windowname')

Names of other widgets are assigned with setWidgetName:

button = gtk. Button()

gtklogger.setWidgetName (button, 'buttonname')

Passive objects like Frames don't need to have names, but it can be convenient to give them names if it will make a log file more readable. Widgets are identified in log files by a colon-separated string of names, starting from a top level widget. For example, 'windowname: frame:button' identifies a widget named 'button' inside a widget named ' frame' inside a top-level widget named 'windowname'. There may be unnamed nested containers in the hierarchy between 'windowname' and 'frame' or between ' frame' and 'button', but the string is a legal identifier for the button as long as there are no other widgets that could answer to the same name. For example, if a window contains a gtk. vBox that contains two gtk. Frames that each contain one gtk. Button, the Buttons can have the same name if the Frames have different names, or the Frames can be unnamed if the Buttons have different names. (If the names cannot be resolved uniquely, errors may occur during playback.)

An advantage of not giving names to intermediate container widgets is that it makes it more likely that reorganizing the GUI will not change the full names of the active widgets, and will not invalidate previously recorded log files. Of course, modifying the interface by removing widgets or replacing one widget type with another will make old log files unreplayable.

\subsubsection{Connecting to Signals}

PyGTK widgets interact with a program by emitting "signals", indicating that their state has been changed (either interactively by the user or programmatically via a function call). A program "connects" to an object's signals if it wants to be notified of changes in the state of the object. For example, the following standard PyGTK code fragment puts a button in a window and calls a callback function, buttoncB, when the button is clicked:

window $=$ gtk. Window ()

button $=$ gtk. Button ('Press Here')

1 gtklogger's widget names are not the same as the names that may be assigned by PyGTK's gtk. Widget. set_name function. Names set by that function are used in GTK resource files for assigning styles to widgets, and need not be unique. gtklogger names must uniquely identify a widget. 
window.add (button)

button.connect ('clicked', buttonCB)

gtklogger defines a new function, gtklogger. connect, which works like the gtk connect function, but also connects to a callback that logs events. ${ }^{2}$ When using gtklogger, the above code snippet looks like this:

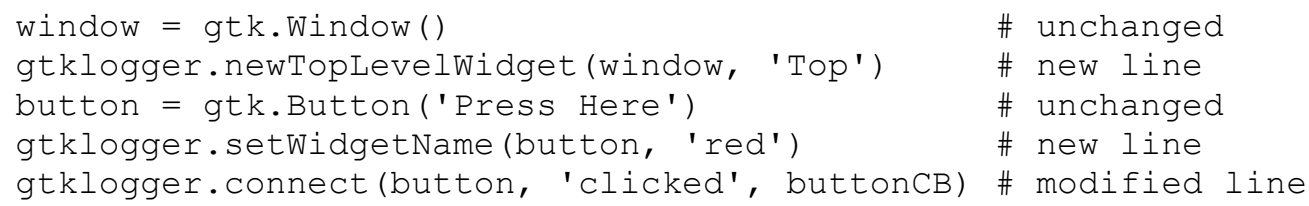

Each time the button is clicked, the line

findWidget ('Top:red') . clicked ()

will be recorded in the log file, and buttonCB will be called. During playback, the line will be read, the gtklogger function findWidget will retrieve the named button, and the mouse click will be simulated by calling the button's clicked method.

Sometimes events that don't have callbacks need to be recorded and replayed. For example, if the appearance of the user interface depends on the window size, it will be necessary for gtklogger to record 'configure-event' signals, even though the program might not otherwise catch that signal. gtklogger defines a connect_passive function to do this, like this:

window $=$ gtk. Window (gtk.WINDOW_TOPLEVEL)

gtklogger.newTopLevelWidget (win̄dow, 'windowname')

gtklogger.connect_passive(window, 'configure-event')

connect_passive works just like connect, except that it doesn't take a callback function argument.

When widgets have their state set by the program, rather than by a user, they generally should not call their callbacks, and should definitely not record their change of state in the gtklogger log file. This is because the log file records user actions. If a user action indirectly makes a widget change state, and if that change of state is recorded in the log file, then when the log is replayed the change of state will occur twice: once in response to the log entry for the original user action, and once for the log entry of the state change. Therefore it's necessary for logging to be suppressed before the program changes the state of a GObject. In normal operation, gtk+ signals are blocked and unblocked by calling an object's handler_block and handler_unblock methods, which take as an argument the return value of the corresponding connect call. Because gtklogger. connect actually makes two connections (one to the

${ }^{2}$ Ideally, gtklogger would simply redefine the connect method for all PyGTK classes, but PyGTK doesn't allow this. It's necessary instead to call a gtklogger function explicitly. 
callback and one to the logging mechanism), gtklogger provides an easy way of blocking both connections at once. gklogger.connect and gtklogger.connect_passive return a GUISignals object that has its own block and unblock methods. For example, the following snippet sets the state of a button without logging it:

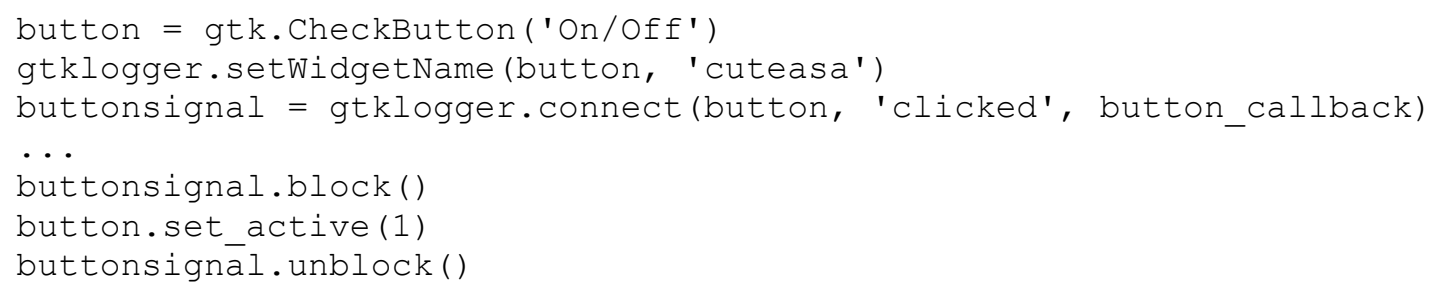

Cautious programmers will use a try: ... except: block to ensure that unblock is called even if an exception occurs when setting the button state. If it's necessary for some reason to block logging, but still execute the callback function, use block_log and unblock_log instead of block and unblock.

\subsubsection{Adopting Non-Widgets}

gtklogger's method for identifying objects in the log file only works for gtk. Widgets, because it relies on the parent/child relationship that widgets have with their containers. However, there are other objects, derived from the gtk. Widget base class, gobject. Gobject, that also emit signals that must be logged. The gtk. Adjustments that underlie scrollbars and sliders and the gtk. CellRenderers, gtk.TreeModels and gtk. TreeSelections that underlie gtk. TreeViews are examples of non-widgets that emit signals. For these to be logged, they must be adopted by a named widget. Adoption establishes a pseudo-parent/child relationship between a named widget and the non-widget that is to be logged.

The gtklogger module provides the function adoptGobject for this purpose. For example, the following code creates a gtk. TreeView and calls the callback function selectioncB when the TreeView's selection changes:

tv $=$ gtk. TreeView $(\ldots)$

selection = tv.get_selection()

selection.connect (''changed', selectionCB)

To log the changes, the TreeView must be named, the Treeselection must be adopted, and the change signal must be logged:

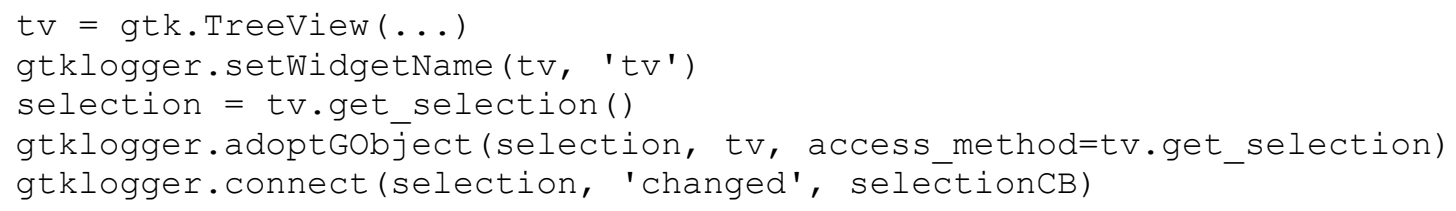

The first two arguments to adoptGObject are the adoptee and the adoptive parent. The third argument is a keyword argument which must be either access_method (specifying a member function of the parent 
that can be called to retrieve the adoptee) or access_function (specifying a non-member function ${ }^{3}$ with the same purpose). There are two optional keyword arguments, a tuple access_args and a dictionary access_kwargs, which provide additional positional and keyword arguments to be passed to the access function.

gtklogger also provides a few convenience functions to facilitate the adoption process. logScrollBars (window, name) sets up logging for the scroll bars of the given gtk. Scrolledwindow object. gtklogger. findCellRenderer can be used as the access function when adopting a gtk. CellRenderer used in a gtk. TreeView. For details, please see the comments in the gtklogger source code (found in core.py and logutils.py).

\subsubsection{Menus and Menu Items}

gtk. MenuItems in gtklogger are handled like other widgets - they must have names and must be connected with gtklogger. connect - but they do have one additional requirement: submenus must be assigned to menu items with

gtklogger.set_submenu(menuitem, menu)

instead of with

menuitem.set_submenu(menu).

If a gtk. Menu is being used as a popup menu, gtklogger. newTopLevelWidget should be use to declared it to be a top level widget, and its 'deactivate' signal should be logged with gtklogger.connect_passive.

\subsubsection{Dialogs}

Dialog boxes need special treatment in gtklogger because the replay mechanism needs extra clues to handle them properly. All calls to gtk. Dialog must therefore be replaced by gtklogger. Dialog. The gtklogger version is derived from the gtk version, and redefines its__init_, run, and add_button methods.

gtklogger at present does not redefine the gtk classes that derive from gtk. Dialog, such as gtk.MessageDialog. In order to use these classes, it will be necessary to create gtklogger variants of them, incorporating the gtklogger. Dialog modifications (from the file gtklogger/core.py).

\subsubsection{Checkpoints}

So far we've discussed four modifications that must be made to a PyGTK program in order to log and replay sessions with gtklogger. If the program uses threads, one more set of modifications needs to be made.

Consider a program in which a button click triggers a long calculation on a separate thread. After the calculation finishes, the user clicks on another button. If there are no other user events between the button clicks, the log file will simply contain two consecutive button click lines. While replaying (see

${ }^{3}$ If a non-member access function is used, it must be inserted into gtklogger's namespace by calling gtklogger.replayDefine so that it may be located when replaying. 
Sec. Table 1: The PyGTK objects that gtklogger currently handles, and the signals that can be logged for each. The list can be extended by defining new GtkLogger subclasses.

3.2), gtklogger has no way to know that the second button click shouldn't be performed until after the long calculation finishes. The thread reading the log file will execute the second button click immediately after the first, with possibly unexpected results.

The solution to synchronizing the playback of a multithread program's log file is to add checkpoints to the program. When recording a session, each call the application makes to

gtklogger.checkpoint $(x)$ inserts a line in the log file. The argument $x$ is a string identifying the checkpoint. When replaying a log file, gtklogger won't proceed past a checkpoint in the file until the corresponding checkpoint in the program has been reached. In the example above, calling gtklogger. checkpoint ('whew') at the end of the long calculation will insert a checkpoint into the log file between the two button-click lines, which will prevent the second click from being simulated until the calculation is done. The log file will look something like this:

findWidget ('window: ... :button1').clicked()

checkpoint whew

findWidget ('window: . . . :button2').clicked()

Checkpoints have identifying labels because programs will typically contain many checkpoints and may execute many simultaneous threads. During playback, gtklogger keeps track of how many times, $L_{x}$, each checkpoint label $x$ has been seen in the log file and how many times, $P_{x}$, checkpoint (x) has been called by the program. Each time a checkpoint $x$ is read from the log, no further log lines will be read until $L_{x} \leq P_{x}$.

Note that, if two or more threads are both using checkpoints, this scheme is insensitive to the order in which the checkpoints are reached. It also never requires the program itself to stop and wait when it reaches a checkpoint - only the replay mechanism waits. This is important, because it means that new checkpoints can be added to a program without invalidating existing log files. The log files don't include the new checkpoints, so the replay mechanism will not wait for them. If it's necessary to add new checkpoints to an existing log file, the log file can be rerecorded while it is being replayed (see Sec. 2.2 and Table 1: The PyGTK objects that gtklogger currently handles, and the signals that can be logged for each. The list can be extended by defining new GtkLogger subclasses.

\section{$3.2)$.}

If it's necessary to remove checkpoints from a program, however, it's important to update the log files. Extra checkpoints in a log file will halt the replay mechanism. If a checkpoint with a given label has been completely eliminated from a program, it's simple to remove all of its lines from a log file using a text editor. If instead, the program flow has just been modified so that a checkpoint is reached fewer times than it had been before the modification, it can be tricky to figure out which checkpoint lines need to be removed from the log file. To make this easier, calls to checkpoint_count () can be inserted manually into the log file. checkpoint_count () takes two arguments: the checkpoint label (a character string) and an integer count. The count argument is optional, and defaults to zero.

checkpoint_count () will raise an exception if the named checkpoint has not been reached in the code exactly count times more than it has been reached in the log file; that is, it asserts that $P_{x}-L_{x}=$ count. 
Checkpoints are also useful simply as a way of marking locations in log files, so that tests can be inserted at the appropriate points. See Sec. 2.3.

\subsection{Recording and Replaying a Session}

To begin recording a session, the program must call gtklogger. start (). The first argument to start () must be the name of the log file. The remaining three arguments are optional keyword arguments. debugLevel is an integer that controls debugging output (the default value is 2, which causes all log lines to be echoed to the terminal, as well as reporting signals which can't be logged for some reason). The second optional argument, suppress_motion_events, is discussed in Sec. 2.4.1. It can usually be omitted. The third, logger_comments, is a boolean that is discussed in Sec. 2.3.

Only one log file can be open for writing at a time. If start () is called while another file is open, the open file will be closed automatically.

To stop recording a session, the program must simply call gtklogger. stop ().

To load and replay a log file, the program must call gtklogger.replay(). This is the step that tests whether or not the program is working correctly. In the simplest case, the program can pass a test just by replaying the entire log file without crashing, raising an exception, or otherwise terminating abnormally. In most cases, however, it will be necessary to add additional tests to the log file, as described in Sec.2.3.

replay () takes a number of arguments, all but one of which are optional. The required argument is the name of the log file which is to be loaded. The optional arguments are

- beginCB: a callback function that is called after the replay machinery is initialized, but before any lines are read from the log file. The function does not have any arguments. The default value is None.

- finishCB: a callback function that is called after the last line is read from the log file. The function does not have any arguments. The default value is None.

- debugLevel: an integer that determines the amount of debugging output. The default value is 2, with the same meaning as in the run () command.

- threaded: a boolean indicating whether or not the program uses threads. If the program is threaded, this parameter must be set to True. The default value is False.

- exceptHook: a function that is called if the replay mechanism catches an exception while executing a line from the log file. The exception is passed in as an argument. The function should return True if it has handled the exception, and False if the exception should be propagated further. In both cases, no further lines will be read from the log file. The default value of exceptHook is None.

- rerecord: the name of a file in which to save the log data, just as if the session were being recorded with gtklogger. start (rerecord). Re-recording a file can be a quick way of incorporating new checkpoints into an old log. When re-recording, comments and assert 
statements in the old $\log$ are copied verbatim into the new log, but the assertions are not executed. ${ }^{4}$ The default value of rerecord is None.

- checkpoints: a boolean value indicating if checkpoints should be used when executing the log file. It can sometimes be useful, especially when re-recording, to ignore checkpoints. Usually, though, this causes more problems than it solves. The default value is True, indicating that checkpoints will be respected.

\subsection{Instrumenting a Log File}

After a program has been instrumented and a log file has been recorded, sometimes simply replaying the $\log$ file is sufficient to test that the program is working correctly. More often, though, it will be necessary to run some extra tests while replaying the log. This is done by adding lines to the log file, using any text editor.

gtklogger log files are mostly, but not quite, Python code. They are read one line at a time ${ }^{5}$, and most lines are passed to the Python interpreter. Long lines can be broken by ending them with a backslash $(\backslash)$. One difference between a log file and a real Python script is that code blocks are not allowed in log files, because they can't be processed one line at a time. Another difference between log files and Python scripts is that log files contain special lines that begin with a few non-Python keywords, namely checkpoint, pause, and postpone. These lines aren't processed by the Python interpreter. checkpoint lines are inserted by the gtklogger checkpoint mechanism, as described in Sec. 2.1.6. pause lines, of the form pause $\mathrm{xxx}$ where $\mathrm{xxx}$ is a number, can be inserted by hand, and instruct the playback mechanism to pause for the given number of milliseconds before proceeding to the next line. (checkpoints are generally much more useful than pauses.) postpone lines are inserted automatically by a few kinds of widgets, and should not be modified or inserted manually.

Like real Python scripts, log files can contain comments preceded by \#.

The prohibition against code blocks in log files doesn't seriously limit the kinds of testing that can be done, because import statements are allowed, so a log file can contain lines like this:

import mytests

assert mytests.testX() $==1$

which will raise an exception if mytests.testx does not return 1 . Tests should always take the form of assert statements - tests should generally not be run while rerecording (see Sec. 2.2), and assert statements in the log file are skipped in that context.

Tests should be inserted into the log file wherever it's necessary to check that the user interface or any other program component is in a particular state. Finding the most useful locations in the log file for tests can be difficult, but some strategies are useful:

\footnotetext{
${ }^{4}$ This is because log files often have to be rerecorded when something is wrong with the program, and assertions can make the program fail before the log file has been completely re-recorded.

${ }^{5}$ Processing one line at at time allows synchronization with the gtk event loop. Python code in $\log$ files is not evaluated until gtk requires input.
} 
1. Add comments to the log file as it is being recorded, indicating what tests should be done. To make this easy to do, if the optional logger_comments argument to gtklogger.start() is True, then instead of writing the log file directly, gtklogger will pipe its output through a primitive GUI which allows the user to type comments into the log file. The GUI is written in PyGTK and runs in a separate process so that it won't interfere with gtklogger. The default value of

logger_comments is True.

In principle, anything could be added to the log file in this way, including assert statements. In practice, it is often easier to instrument a log file after recording it completely.

2. Look for log lines referring to named widgets. Any line that simulates an action of a particular Widget will begin with a call to findWidget (...), where the argument is a colon-separated string containing the name of the widget and its parent containers. Menu item activation corresponds to lines beginning with calls to findMenu (...).

3. Use checkpoints. If the program calls gtklogger. checkpoint () after performing some action, the tests to make sure that the action has been performed correctly should be inserted in the log file just after the checkpoint. See 2.1.6.

4. Insert an assert False line in the log file, which will cause replaying to halt at that point in the file, at which time it's possible to examine the state of the interface and figure out what's happening there. This only works if the user interface is known to be working correctly, and if an exceptHook is being used to prevent the program from terminating with a Python

AssertionError.

gtklogger contains a few routines designed to make it easier to write functions that test the state of the user interface.

- gtklogger.findWidget (name) returns the PyGTK Widget with the specified name, which is a colonseparated string identifying the Widget, as discussed in Sec. 2.1.1.

- gtklogger.findMenu(menushell, path) returns a gtk. MenuItem contained in the given gtk.MenuShell. MenuShell is a Widget, so it should be named and located by gtklogger.findWidget. path is a colon-separated string identifying the menu item by its name and the names of its parent menus. For example, if the gtklogger name of a gtk. MenuBar is window: menubar, then the Quit item in the File menu would be returned by

findMenu (findWidget ('window:menubar'), 'File:Quit')

- gtklogger.findAllWidgets (top) prints out the names of all named widgets whose colon-separated string names begin with top. It won't find adopted non-widgets. This is useful for debugging, and to find the full name of a widget whose name you've forgotten.

\subsection{Miscellaneous Functions}

\subsubsection{Motion Events}

If a callback is connected to a Widget's motion-notify-event, every mouse motion within the widget will be logged. Often, this will create a whole blizzard of lines in the log file, most of which aren't actually necessary. gtklogger contains two functions, log_motion_events () and 
dont_log_motion_events(), which can be called to turn motion logging on and off, so that only interesting motions are recorded. The initial status is controlled by the optional suppress_motion_events keyword argument to the start() function, whose default value is True.

If log_motion_events and dont_log_motion_events are passed a gtk.widget argument, then they apply only to motion events within that Widget or its children. If they're called with no argument, they apply to all widgets, erasing any previous widget-specific settings.

\subsubsection{Exceptions}

If a program raises exceptions that are not derived from the Python Exception base class, gtklogger should be notified about those exceptions by calling gtklogger.add_exception(). The argument to add_exception is the class of the expected exceptions. The function should be called before replaying $\log$ files.

\subsubsection{Sanity Checking}

The function gtklogger.sanity_check (widget) is a debugging tool that checks that the name assigned to a widget correctly identifies it. If two or more widgets have the same name (and their parents all have the same names), then sanity_check will raise an exception for at least one of them. If the given widget is a Container, the check is run on the container and all of its children.

The function gtklogger.comprehensive_sanity_check() runs the sanity check on all known named widgets.

\section{Details}

This section discusses some details of the gtklogger implementation that aren't necessary for users to know, but are required reading for those who wish to extend or modify it.

\subsection{Loggers}

When a PyGTK object emits a signal, as long as that signal has been connected with gtklogger.connect or gtklogger.connect_passive, gtklogger creates an instance of a GtkLogger subclass, and uses that instance's record method to store the event in the log file. The GtkLogger class hierarchy mirrors (more or less) the PyGTK class hierarchy. If a GtkLogger instance can't handle a particular signal, it passes it on to its parent class's record method. If the signal is passed all the way back to the GtkLogger base class, an exception is raised.

Each GtkLogger subclass must have a record ( ) method, taking the arguments self, object, signal, and *args. object is the PyGTK object that emitted the signal, and signal is the signal it emitted. If the signal's callback signature includes extra arguments, these are contained in *args. GtkLogger.record's job is to return a list of strings which are to be written into the log file, one per line. These lines are Python code that, when executed by gtklogger.replay, will reproduce the event that triggered the signal. Those lines will have to contain information about which PyGTK object emitted the signal. To get that information, record should call GtkLogger.location (object, *args) with the same object and *args that were passed in as arguments. location returns a string containing the appropriate gtklogger. findWidget invocation for the PyGTK object.

All GtkLogger subclasses are actually derived from two intermediate classes, gtklogger.widgetlogger.WidgetLogger and gtklogger. adopteelogger. AdopteeLogger. These two classes take care of defining the location functions for all other subclasses. 
Besides having record and location methods, GtkLogger subclasses must contain a tuple called classes that lists the PyGTK classes which they can log. When the gtklogger signal handler creates a logger for a PyGTK object, it looks for the GtkLogger class that lists the most derived base class for the PyGTK object. That is, the GtkLogger subclass that includes gtk. Button in its classes tuple will be used to log all gtk. Button objects and all objects in subclasses of gtk. Button, unless those subclasses are explicitly listed in another GtkLogger's classes tuple. 
As a concrete example, consider the simple gtk. Button. It's GtkLogger looks like this:

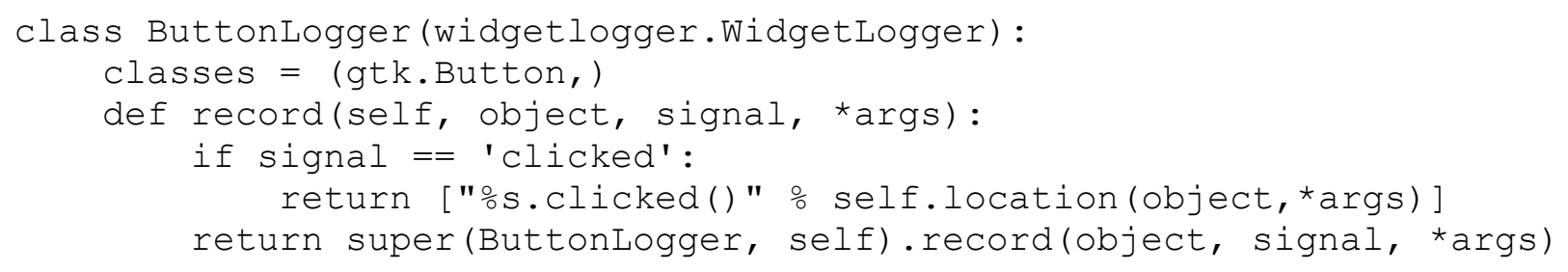

The record method handles only the ' clicked' signal. It returns a line that, when executed, will call the button's clicked () method, which is the programmatic equivalent of pressing the button. The call to self.location() generates code that ensures that the correct button is virtually clicked. Finally, in case the signal is something other than ' $\mathrm{Clicked}$, the final line calls the base class record function. This is why the GtkLogger class hierarchy mimics the PyGTK class hierarchy — signals handled in PyGTK by base classes are logged by GtkLogger base classes.

The GtkLogger for gtk. Adjustment objects looks like this:

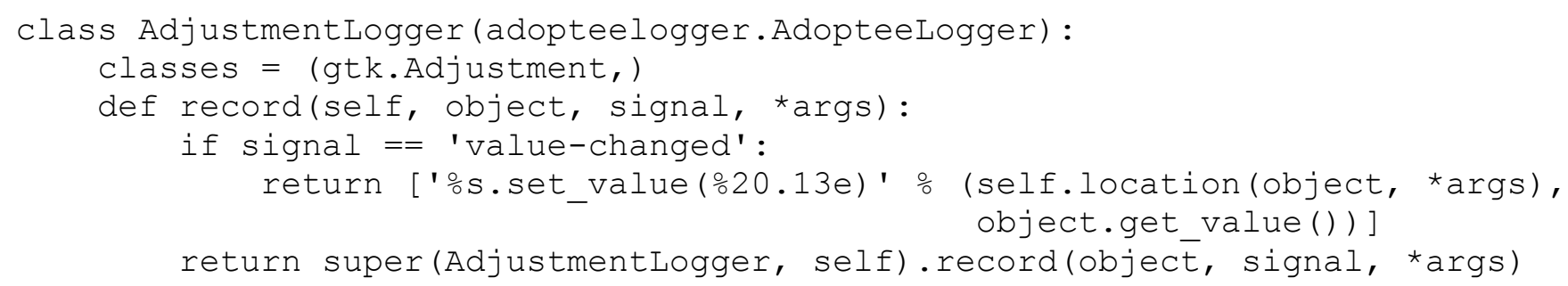

Adjustments aren'twidgets, so their GtkLogger is derived from AdopteeLogger instead of WidgetLogger. Nonetheless, the same call to self.location() generates the code that will identify the Adjustment while replaying. The 'value-changed' event will be recreated by calling the Adjustment's set_value() method with its current value.

The gtklogger package only includes GtkLoggers for the PyGTK objects and signals that we've used in our programs [3]. These are listed in Table Error! Reference source not found.. Users who extend this list by adding new subclasses or new signals are invited to contribute them via the gtklogger web page or by sending an e-mail to the authors.

A few utility functions are provided for use in GtkLogger's record functions.

If it's necessary for a GtkLogger to use a local variable in the multiline output of a record function, the function loggers. localvar (basename) should be used to acquire a unique variable name. The name will be the string given by basename, plus_ $\mathrm{xxx}$ where $\mathrm{xxx}$ is an integer.

A line in a log file can create a gtk. gdk. Event object by calling gtklogger.event (). The first argument to event must be an event type, such as gtk.gdk.BUTTON_PRESS. The remaining arguments are keyword arguments specifying attributes of the Event. 


\begin{tabular}{|c|c|}
\hline PyGTK object & signals \\
\hline Widget & $\begin{array}{l}\text { focus-in-event focus-out-event } \\
\text { size-allocate button-press-event button- } \\
\text { release-event motion-notify-event }\end{array}$ \\
\hline Adjustment & value-changed \\
\hline Button & clicked \\
\hline RadioButton & clicked \\
\hline ComboBox & changed \\
\hline Entry & changed \\
\hline Expander & activate \\
\hline Paned & notify: :position \\
\hline MenuItem & activate \\
\hline MenuShell & cancel deactivate \\
\hline TreeView & $\begin{array}{l}\text { button-release-event row-activated } \\
\text { row-expanded row-collapsed }\end{array}$ \\
\hline Treeselection & changed \\
\hline Liststore & row-inserted row-deleted \\
\hline CellRenderer & toggled \\
\hline Window & destroy delete-event configure-event \\
\hline
\end{tabular}

Table 1: The PyGTK objects that gtklogger currently handles, and the signals that can be logged for each. The list can be extended by defining new GtkLogger subclasses.

\subsection{The Replay Mechanism}

At its core, the gtklogger replay mechanism is conceptually simple, but is made complicated by a few pesky details. When replaying a log file, gtklogger reads the file and creates a GUILogLineRunner instance for each line in the file. There are different kinds of GUILogLineRunners for different types of lines (code, checkpoints, comments, etc.). GUILogLineRunners have a_call_method that executes the line. Each GUILogLineRunner is installed as a PyGK idle callback so that the log file lines can be executed without affecting the basic control loops of the program. Idle callbacks are executed by the gtk event loop when it has no other tasks to perform.

One complication arises because the gtk event loop can be re-entrant: a function called by the event loop can itself invoke the event loop to process events. For example, when an event causes a modal dialog box to appear, the dialog box will process events in its own event loop until the box is closed. The nested event loops make it possible for the processing of one gtk event to start before the processing of the previous one has completed. The event handler that creates a dialog box may not return until the dialog box is closed, but the box can't be closed unless the event handler for its OK button is run. Therefore it's necessary for further events to be handled before the handler for the initial event is complete.

For gtklogger, this means that care must be taken to ensure that the idle callbacks for log file lines run at the correct times. Because one GUILogLineRunner may have to run before the previous one has 
finished, when a GUILogLineRunner is run, it first installs the next GUILogLineRunner as an idle callback. It then checks to see if the previous line has either completed or is running in a less deeply nested event loop. Only then does it actually execute its own code. If the previous line isn't ready, the current line reinstalls itself as a time-out callback, which schedules it to be run again after a specified delay $^{6}$. (This is the reason that a program using gtklogger has to replace all gtk. Dialogs with gtklogger. Dialogs. The gtklogger version contains code for monitoring the nesting depth of the event loop.)

When a checkpoint line is encountered, gtklogger compares the number of times the checkpoint's label has been seen in the log file and the number of times that the same label has been passed to checkpoint functions in the code (as described in Sec. 2.1.6). If replaying has to wait, then the current GUILogLineRunner installs itself as a PyGTK time-out callback, which means that it will run again after a specified interval (100 ms, by default). It will do this repeatedly until the checkpoint is satisfied, at which point it will indicate that it has finished, allowing the next line in the log file to run.

\section{Effectiveness}

The gtklogger tool described here was developed principally to assist with testing the OOF [3] project, and all of our experience with it is in this environment.

The nature of the tests implemented using this tool is somewhat ad-hoc, as is typical of real-world software project where testing is introduced part way through the development process. Consequently, the primary benefit to the OOF project has been to ensure that the functionality for which tests have been written - necessarily that which is thought to be important by the development team - continues to pass the tests as development continues. In this way, the GUI test suite has had the character of a regression test set. In addition to ensuring that correct functionality stays correct, we have written tests for GUI bugs once they are discovered, to ensure that, once fixed, GUI bugs also stay fixed.

A more thorough testing scheme could use this tool to implement test cases generated from user feedback or from other automatic tools. Extending the GUI tests to do this is a topic of current research.

\section{Obtaining gtklogger}

gtklogger may be downloaded free of charge from

http://www.ctcms.nist.gov/oof/gtklogger/

That page includes the source code, this paper, and a demonstration program.

gtklogger was produced by NIST, an agency of the U.S. government, and by statute is not subject to copyright in the United States. Recipients of the software assume all responsibilities associated with its operation, modification and maintenance.

\section{Acknowledgments}

Rhonald Lua and Edwin García were supported in part by the National Science Foundation through the Information Technology Research Grant DMR-0205232.

\section{References}

[1] http://www.gtk.org.

\footnotetext{
${ }^{6}$ The delay is set to $100 \mathrm{~ms}$ by default.
} 
[3] S. A. Langer, A. C. E. Reid, R. E. García, S.-I. Haan, R. C. Lua, W. C. Carter, E. R. Fuller, Jr, and A. Roosen, OOF: Analysis of real material microstructures, [Web page], http://www.ctcms.nist.gov/oof/index.html [accessed 4/8/15].

[4] J. Andersson and G. Bache, Extreme Programming and Agile Processes in Software Engineering (Springer Berlin/Heidelberg, 2004), vol. 3092 of Lecture Notes in Computer Science, chap. The Video Store Revisited Yet Again: Adventures in GUI Acceptance Testing, pp. 1-10. 